\title{
Higher order Hermite-Fejér interpolation polynomials with Laguerre-type weights
}

\author{
Heesun Jung ${ }^{1 *}$ and Ryozi Sakai
}

\author{
* Correspondence: hsun90@skku. \\ edu \\ ${ }^{1}$ Department of Mathematics \\ Education, Sungkyunkwan \\ University Seoul 110-745, Republic \\ of Korea \\ Full list of author information is \\ available at the end of the article
}

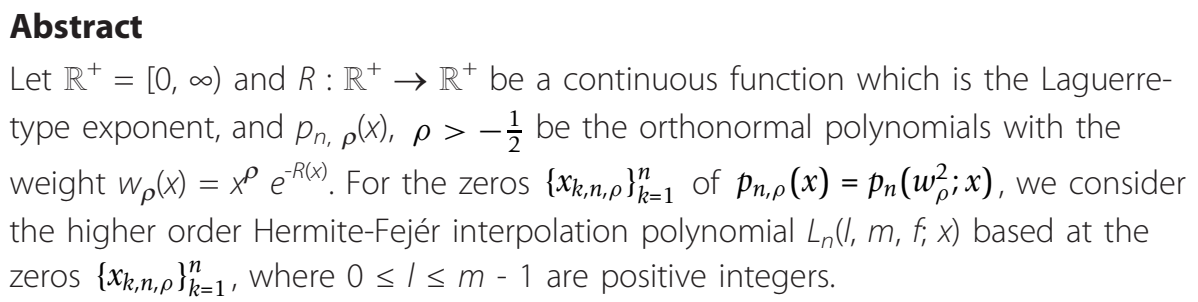

Keywords: Laguerre-type weights, orthonormal polynomials, higher order HermiteFejér interpolation polynomials

\section{Introduction and main results}

Let $\mathbb{R}=[-\infty, \infty)$ and $\mathbb{R}^{+}=[0, \infty)$. Let $R: \mathbb{R}^{+} \rightarrow \mathbb{R}^{+}$be a continuous, non-negative, and increasing function. Consider the exponential weights $w_{\rho}(x)=x^{\rho} \exp (-R(x)), \rho>-1 / 2$, and then we construct the orthonormal polynomials $\left\{p_{n, \rho}(x)\right\}_{n=0}^{\infty}$ with the weight $w_{\rho}$ $(x)$. Then, for the zeros $\left\{x_{k, n, \rho}\right\}_{k=1}^{n}$ of $p_{n, \rho}(x)=p_{n}\left(w_{\rho}^{2} ; x\right)$, we obtained various estimations with respect to $p_{n, \rho}^{(j)}\left(x_{k, n, \rho}\right), k=1,2, \ldots, n, j=1,2, \ldots, v$, in [1]. Hence, in this article, we will investigate the higher order Hermite-Fejér interpolation polynomial $L_{n}(l$, $m, f ; x)$ based at the zeros $\left\{x_{k, n, \rho}\right\}_{k=1}^{n}$, using the results from [1], and we will give a divergent theorem. This article is organized as follows. In Section 1, we introduce some notations, the weight classes $\mathcal{L}_{2}, \tilde{\mathcal{L}}_{v}$ with $\mathcal{L}\left(C^{2}\right), \mathcal{L}\left(C^{2}+\right)$, and main results. In Section 2, we will introduce the classes $\mathcal{F}\left(C^{2}\right)$ and $\mathcal{F}\left(C^{2}+\right)$, and then, we will obtain some relations of the factors derived from the classes $\mathcal{F}\left(C^{2}\right), \mathcal{F}\left(C^{2}+\right)$ and the classes $\mathcal{L}\left(C^{2}+\right), \mathcal{L}\left(C^{2}+\right)$. Finally, we will prove the main theorems using known results in [1-5], in Section 3.

We say that $f: \mathbb{R} \rightarrow \mathbb{R}^{+}$is quasi-increasing if there exists $C>0$ such that $f(x) \leq C f(y)$ for $0<x<y$. The notation $f(x) \sim g(x)$ means that there are positive constants $C_{1}, C_{2}$ such that for the relevant range of $x, C_{1} \leq f(x) / g(x) \leq C_{2}$. The similar notation is used for sequences, and sequences of functions. Throughout this article, $C, C_{1}, C_{2}$, ... denote positive constants independent of $n, x, t$ or polynomials $P_{n}(x)$. The same symbol does not necessarily denote the same constant in different occurrences. We denote the class of polynomials with degree $n$ by $\mathcal{P}_{n}$.

(C) 2011 Jung and Sakai; licensee Springer. This is an Open Access article distributed under the terms of the Creative Commons Attribution License (http://creativecommons.org/licenses/by/2.0), which permits unrestricted use, distribution, and reproduction in any medium, provided the original work is properly cited. 
First, we introduce classes of weights. Levin and Lubinsky $[5,6]$ introduced the class of weights on $\mathbb{R}^{+}$as follows. Let $I=[0, d)$, where $0<d \leq \infty$.

Definition 1.1. $[5,6]$ We assume that $R: I \rightarrow[0, \infty)$ has the following properties: Let $Q(t)=R(x)$ and $x=t^{2}$.

(a) $\sqrt{x} R(x)$ is continuous in $I$, with limit 0 at 0 and $R(0)=0$;

(b) $R^{\prime \prime}(x)$ exists in $(0, d)$, while $Q^{\prime \prime}$ is positive in $(0, \sqrt{d})$;

(c)

$$
\lim _{x \rightarrow d-} R(x)=\infty
$$

(d) The function

$$
T(x):=\frac{x R^{\prime}(x)}{R(x)}
$$

is quasi-increasing in $(0, d)$, with

$$
T(x) \geq \Lambda>\frac{1}{2}, \quad x \in(0, d)
$$

(e) There exists $C_{1}>0$ such that

$$
\frac{\left|R^{\prime \prime}(x)\right|}{R \prime(x)} \leq C_{1} \frac{R^{\prime}(x)}{R(x)}, \quad \text { a.e. } \quad x \in(0, d) .
$$

Then, we write $w \in \mathcal{L}\left(C^{2}\right)$. If there also exist a compact subinterval $J^{*} \ni 0$ of $I^{*}=(-\sqrt{d}, \sqrt{d})$ and $C_{2}>0$ such that

$$
\frac{Q^{\prime \prime}(t)}{\left|Q^{\prime}(t)\right|} \geq C_{2} \frac{\left|Q^{\prime}(t)\right|}{Q(t)}, \quad \text { a.e. } \quad t \in I^{*} \backslash J^{*},
$$

then we write $w \in \mathcal{L}\left(C^{2}+\right)$.

We consider the case $d=\infty$, that is, the space $\mathbb{R}^{+}=[0, \infty)$, and we strengthen Definition 1.1 slightly.

Definition 1.2. We assume that $R: \mathbb{R}^{+} \rightarrow \mathbb{R}^{+}$has the following properties:

(a) $R(x), R^{\prime}(x)$ are continuous, positive in $\mathbb{R}^{+}$, with $R(0)=0, R^{\prime}(0)=0$;

(b) $R^{\prime \prime}(x)>0$ exists in $\mathbb{R}^{+} \backslash\{0\}$;

(c)

$$
\lim _{x \rightarrow \infty} R(x)=\infty
$$

(d) The function

$$
T(x):=\frac{x R^{\prime}(x)}{R(x)}
$$


is quasi-increasing in $\mathbb{R}^{+} \backslash\{0\}$, with

$$
T(x) \geq \Lambda>\frac{1}{2}, \quad x \in \mathbb{R}^{+} \backslash\{0\}
$$

(e) There exists $C_{1}>0$ such that

$$
\frac{R^{\prime \prime}(x)}{R^{\prime}(x)} \leq C_{1} \frac{R^{\prime}(x)}{R(x)}, \quad \text { a.e. } \quad x \in \mathbb{R}^{+} \backslash\{0\} .
$$

There exist a compact subinterval $J \ni 0$ of $\mathbb{R}^{+}$and $C_{2}>0$ such that

$$
\frac{R^{\prime \prime}(x)}{R^{\prime}(x)} \geq C_{2} \frac{R^{\prime}(x)}{R(x)}, \quad \text { a.e. } \quad t \in \mathbb{R}^{+} \backslash
$$

then we write $w \in \mathcal{L}_{2}$.

To obtain estimations of the coefficients of higher order Hermite-Fejér interpolation polynomial based at the zeros $\left\{x_{k, n, \rho}\right\}_{k=1}^{n}$, we need to focus on a smaller class of weights.

Definition 1.3. Let $w=\exp (-R) \in \mathcal{L}_{2}$ and let $v \geq 2$ be an integer. For the exponent $R$, we assume the following:

(a) $R^{(j)}(x)>0$, for $0 \leq j \leq v$ and $x>0$, and $R^{(j)}(0)=0,0 \leq j \leq v-1$.

(b) There exist positive constants $C_{i}>0, i=1,2, \ldots, v-1$ such that for $i=1,2, \ldots$, $v-1$

$$
R^{(i+1)}(x) \leq C_{i} R^{(i)}(x) \frac{R^{\prime}(x)}{R(x)}, \quad \text { a.e. } \quad x \in \mathbb{R}^{+} \backslash\{0\} .
$$

(c) There exist positive constants $C, c_{1}>0$ and $0 \leq \delta<1$ such that on $x \in\left(0, c_{1}\right)$

$$
R^{(v)}(x) \leq C\left(\frac{1}{x}\right)^{\delta}
$$

(d) There exists $c_{2}>0$ such that we have one among the following

(d1) $T(x) / \sqrt{x}$ is quasi-increasing on $\left(c_{2}, \infty\right)$,

(d2) $R^{(v)}(x)$ is nondecreasing on $\left(c_{2}, \infty\right)$.

Then we write $w(x)=e^{-R(x)} \in \tilde{\mathcal{L}}_{v}$.

Example 1.4. [6,7] Let $v \geq 2$ be a fixed integer. There are some typical examples satisfying all conditions of Definition 1.3 as follows: Let $\alpha>1, l \geq 1$, where $l$ is an integer. Then we define

$$
R_{l, \alpha}(x)=\exp _{l}\left(x^{\alpha}\right)-\exp _{l}(0),
$$

where $\exp _{l}(x)=\exp (\exp (\exp \ldots \exp (x)) \ldots)$ is the $l$-th iterated exponential. 
(1) If $\alpha>v, w(x)=e^{-R_{l, \alpha}(x)} \in \tilde{\mathcal{L}}_{v}$.

(2) If $\alpha \leq v$ and $\alpha$ is an integer, we define

$$
R_{l, \alpha}^{*}(x)=\exp _{l}\left(x^{\alpha}\right)-\exp _{l}(0)-\sum_{j=1}^{r} \frac{R_{l, \alpha}^{(j)}(0)}{j !} x^{j} .
$$

Then $w(x)=e^{-R_{l, \alpha}^{*}(x)} \in \tilde{\mathcal{L}}_{v}$.

In the remainder of this article, we consider the classes $\mathcal{L}_{2}$ and $\tilde{\mathcal{L}}_{v}$; Let $w \in \mathcal{L}_{2}$ or $w \in \tilde{\mathcal{L}}_{v} v \geq 2$. For $\rho>-\frac{1}{2}$, we set $w_{\rho}(x):=x^{\rho} w(x)$. Then we can construct the orthonormal polynomials $p_{n, \rho}(x)=p_{n}\left(w_{\rho}^{2} ; x\right)$ of degree $n$ with respect to $w_{\rho}^{2}(x)$. That is,

$$
\int_{0}^{\infty} p_{n, \rho}(u) p_{m, \rho}(u) w_{\rho}^{2}(u) d u=\delta_{n m}(\text { Kronecker's delta }) \quad n, m=0,1,2, \ldots
$$

Let us denote the zeros of $p_{n, \rho}(x)$ by

$$
0<x_{n, n, \rho}<\cdots<x_{2, n, \rho}<x_{1, n, \rho}<\infty
$$

The Mhaskar-Rahmanov-Saff numbers $a_{v}$ is defined as follows:

$$
v=\frac{1}{\pi} \int_{0}^{1} \frac{a_{v} t R^{\prime}\left(a_{v} t\right)}{\sqrt{t(1-t)}} \mathrm{d} t, \quad v>0
$$

Let $l, m$ be non-negative integers with $0 \leq l<m \leq v$. For $f \in C^{(l)}(\mathbb{R})$, we define the $(l$, $m$ )-order Hermite-Fejér interpolation polynomials $L_{n}(l, m, f ; x) \in \mathcal{P}_{m n-1}$ as follows: For each $k=1,2, \ldots, n$,

$$
\begin{aligned}
& L_{n}^{(j)}\left(l, m, f ; x_{k, n, \rho}\right)=f^{(j)}\left(x_{k, n, \rho}\right), \quad j=0,1,2, \ldots, l \\
& L_{n}^{(j)}\left(l, m, f ; x_{k, n, \rho}\right)=0, \quad j=l+1, l+2, \ldots, m-1 .
\end{aligned}
$$

For each $P \in \mathcal{P}_{m n-1}$, we see $L_{n}(m-1, m, P ; x)=P(x)$. The fundamental polynomials $h_{s, k, n, \rho}(m ; x) \in \mathcal{P}_{m n-1}, k=1,2, \ldots, n$, of $L_{n}(l, m, f ; x)$ are defined by

$$
h_{s, k, n, \rho}(l, m ; x)=l_{k, n, \rho}^{m}(x) \sum_{i=s}^{m-1} e_{s, i}(l, m, k, n)\left(x-x_{k, n, \rho}\right)^{i} .
$$

Here, $l_{k, n, \rho}(x)$ is a fundamental Lagrange interpolation polynomial of degree $n-1$ [[8], p. 23] given by

$$
l_{k, n, \rho}(x)=\frac{p_{n}\left(w_{\rho}^{2} ; x\right)}{\left(x-x_{k, n, \rho}\right) p_{n}^{\prime}\left(w_{\rho}^{2} ; x_{k, n, \rho}\right)}
$$

and $h_{\mathrm{s}, k, n, \rho}(l, m ; x)$ satisfies

$$
h_{s, k, n, \rho}^{(j)}\left(l, m ; x_{p, n, \rho}\right)=\delta_{s, j} \delta_{k, p} \quad j, s=0,1, \ldots, m-1, p=1,2, \ldots, n .
$$


Then

$$
L_{n}(l, m, f ; x)=\sum_{k=1}^{n} \sum_{s=0}^{l} f^{(s)}\left(x_{k, n, \rho}\right) h_{s, k, n, \rho}(l, m ; x) .
$$

In particular, for $f \in C(\mathbb{R})$, we define the $m$-order Hermite-Fejér interpolation polynomials $L_{n}(m, f ; x) \in \mathcal{P}_{m n-1}$ as the $(0, m)$-order Hermite-Fejér interpolation polynomials $L_{n}(0, m, f ; x)$. Then we know that

$$
L_{n}(m, f ; x)=\sum_{k=1}^{n} f\left(x_{k, n, \rho}\right) h_{k, n, \rho}(m ; x)
$$

where $e_{i}(m, k, n):=e_{0, i}(0, m, k, n)$ and

$$
h_{k, n, \rho}(m ; x)=l_{k, n, \rho}^{m}(x) \sum_{i=0}^{m-1} e_{i}(m, k, n)\left(x-x_{k, n, \rho}\right)^{i}
$$

We often denote $l_{k, n}(x):=l_{k, n, \rho}(x), h_{s, k, n}(x):=h_{s, k, n, \rho}(x)$, and $x_{k, n}:=x_{k, n, \rho}$ if they do not confuse us.

Theorem 1.5. Let $w(x)=\exp (-R(x)) \in \mathcal{L}\left(C^{2}+\right)$ and $\rho>-1 / 2$.

(a) For each $m \geq 1$ and $j=0,1, \ldots$, we have

$$
\left|\left(l_{k, n}^{m}\right)^{(j)}\left(x_{k, n}\right)\right| \leq C\left(\frac{n}{\sqrt{a_{2 n}-x_{k, n}}}\right)^{j} x_{k, n}^{-\frac{j}{2}} .
$$

(b) For each $m \geq 1$ and $j=s, \ldots, m-1$, we have $e_{s, s}(l, m, k, n)=1 / s$ ! and

$$
\left|e_{s, j}(l, m, k, n)\right| \leq C\left(\frac{n}{\sqrt{a_{2 n}-x_{k, n}}}\right)^{j-s-\frac{j-s}{2}} x_{k, n}
$$

We remark $\mathcal{L}_{2} \subset \mathcal{L}\left(C^{2}+\right)$.

Theorem 1.6. Let $w(x)=\exp (-R(x)) \in \tilde{\mathcal{L}}_{v}, v \geq 2$ and $\rho>-1 / 2$. Assume that $1+2 \rho$ $-\delta / 2 \geq 0$ for $\rho<-1 / 4$ and if $T(x)$ is bounded, then assume that

$$
a_{n} \leq C n^{2 /(1+\nu-\delta)}
$$

where $0 \leq \delta<1$ is defined in (1.1). Then we have the following:

(a) If $j$ is odd, then we have for $m \geq 1$ and $j=0,1, \ldots, v-1$,

$$
\begin{aligned}
\left|\left(l_{k, n}^{m}\right)^{(j)}\left(x_{k, n}\right)\right| \leq & C\left(\frac{T\left(a_{n}\right)}{\sqrt{a_{n} x_{k, n}}}+R^{\prime}\left(x_{k, n}\right)+\frac{1}{x_{k, n}}\right) \\
& \times\left(\frac{n}{\sqrt{a_{2 n}}-\sqrt{x_{k, n}}}+\frac{T\left(a_{n}\right)}{\sqrt{a_{n}}}\right)^{j-1} x_{k, n} \frac{j-1}{2} .
\end{aligned}
$$


(b) If $j-s$ is odd, then we have for $m \geq 1$ and $0 \leq s \leq j \leq m-1$,

$$
\begin{aligned}
\left|e_{s, j}(l, m, k, n)\right| \leq & C\left(\frac{T\left(a_{n}\right)}{\sqrt{a_{n} x_{k, n}}}+R^{\prime}\left(x_{k, n}\right)+\frac{1}{x_{k, n}}\right) \\
& \times\left(\frac{n}{\sqrt{a_{2 n}}-\sqrt{x_{k, n}}}+\frac{T\left(a_{n}\right)}{\sqrt{a_{n}}}\right)^{j-s-1} x_{k, n} \frac{j-s-1}{2} .
\end{aligned}
$$

Theorem 1.7. Let $0<\varepsilon<1 / 4$. Let $\frac{1}{\varepsilon} \frac{a_{n}}{n^{2}} \leq x_{k, n} \leq \varepsilon a_{n}$. Let $s$ be a positive integer with 2 $\leq 2 s \leq v$. Then under the same conditions as the assumptions of Theorem 1.6, there exists $\mu_{1}(\varepsilon, n)>0$ such that

$$
\left|p_{n, \rho}^{(2 s)}\left(x_{k, n}\right)\right| \leq C \delta(\varepsilon, n)\left(\frac{n}{\sqrt{a_{n}}}\right)^{2 s-1}\left|p_{n}^{\prime}\left(x_{k, n}\right)\right| x_{k, n}^{-\frac{(2 s-1)}{2}}
$$

and $\delta(\varepsilon, n) \rightarrow 0$ as $n \rightarrow \infty$ and $\varepsilon \rightarrow 0$.

Theorem 1.8. [4, Lemma 10] Let $0<\varepsilon<1 / 4$. Let $\frac{1}{\varepsilon} \frac{a_{n}}{n^{2}} \leq x_{k, n} \leq \varepsilon a_{n}$. Let $s$ be a positive integer with $2 \leq 2 s \leq v-1$. Suppose the same conditions as the assumptions of Theorem 1.6. Then

(a) for $1 \leq 2 s-1 \leq v-1$,

$$
\left|\left(l_{k, n}^{m}\right)^{(2 s-1)}\left(x_{k, n}\right)\right| \leq C \delta(\varepsilon, n)\left(\frac{n}{\sqrt{a_{n}}}\right)^{2 s-1} x_{k, n}^{-\frac{2 s-1}{2}},
$$

where $\delta(\varepsilon, n)$ is defined in Theorem 1.7.

(b) there exists $\beta(n, k)$ with $0<D_{1} \leq \beta(n, k) \leq D_{2}$ for absolute constants $D_{1}, D_{2}$ such that the following holds:

$$
\left(l_{k, n}^{m}\right)^{(2 s)}\left(x_{k, n}\right)=(-1)^{s} \phi_{s}(m) \beta^{s}(2 n, k)\left(\frac{n}{\sqrt{a_{n}}}\right)^{2 s} x_{k, n}^{-s}\left(1+\xi_{s}\left(m, \varepsilon, x_{k, n}, n\right)\right)
$$

and $\left|\xi_{s}\left(m, \varepsilon, x_{k, n}, n\right)\right| \rightarrow 0$ as $n \rightarrow \infty$ and $\varepsilon \rightarrow 0$.

Theorem 1.9. [4, (4.16)], [9]Let $0<\varepsilon<1 / 4$. Let $\frac{1}{\varepsilon} \frac{a_{n}}{n^{2}} \leq x_{k, n} \leq \varepsilon a_{n}$. Let s be a positive integer with $2 \leq 2 s \leq m-1$. Suppose the same conditions as the assumptions of Theorem 1.6. Then for $j=0,1,2, \ldots$, there is a polynomial $\Psi_{j}(x)$ of degree $j$ such that $(-1)^{j} \psi_{j}$ $(-m)>0$ for $m=1,3,5, \ldots$ and the following relation holds:

$$
e_{2 s}(m, k, n)=\frac{(-1)^{s}}{(2 s) !} \Psi_{s}(-m) \beta^{s}(2 n, k)\left(\frac{n}{\sqrt{a_{n}}}\right)^{2 s} x_{k, n}^{-s}\left(1+\eta_{s}\left(m, \varepsilon, x_{k, n}, n\right)\right)
$$

and $\left|\eta_{s}\left(m, \varepsilon, x_{k, n}, n\right)\right| \rightarrow 0$ as $n \rightarrow \infty$ and $\varepsilon \rightarrow 0$.

Theorem 1.10. Let $m$ be an odd positive integer. Suppose the same conditions as the assumptions of Theorem 1.6. Then there is a function $f$ in $C\left(\mathbb{R}^{+}\right)$such that for any fixed 
interval $[a, b], a>0$,

$$
\limsup _{n \rightarrow \infty} \max _{a \leq x \leq b}\left|L_{n}(m, f ; x)\right|=\infty .
$$

\section{Preliminaries}

Levin and Lubinsky introduced the classes $\mathcal{L}\left(C^{2}\right)$ and $\mathcal{L}\left(C^{2}+\right)$ as analogies of the classes $\mathcal{F}\left(C^{2}\right)$ and $\mathcal{F}\left(C^{2}+\right)$ defined on $I^{*}=(-\sqrt{d}, \sqrt{d})$. They defined the following:

Definition 2.1. [10] We assume that $Q: I^{*} \rightarrow[0, \infty)$ has the following properties:

(a) $Q(t)$ is continuous in $I^{*}$, with $Q(0)=0$;

(b) $Q^{\prime \prime}(t)$ exists and is positive in $I^{*} \backslash\{0\}$;

(c)

$$
\lim _{t \rightarrow \sqrt{d}-} Q(t)=\infty ;
$$

(d) The function

$$
T^{*}(t):=\frac{t Q^{\prime}(t)}{Q(t)}
$$

is quasi-increasing in $(0, \sqrt{d})$, with

$$
T^{*}(t) \geq \Lambda^{*}>1, \quad t \in I^{*} \backslash\{0\} ;
$$

(e) There exists $C_{1}>0$ such that

$$
\frac{Q^{\prime \prime}(t)}{\left|Q^{\prime}(t)\right|} \leq C_{1} \frac{\left|Q^{\prime}(t)\right|}{Q(t)}, \quad \text { a.e. } \quad t \in I^{*} \backslash\{0\} .
$$

Then we write $W \in \mathcal{F}\left(C^{2}\right)$. If there also exist a compact subinterval $\digamma^{*} \ni 0$ of $I^{*}$ and $C_{2}>0$ such that

$$
\frac{Q^{\prime \prime}(t)}{\left|Q^{\prime}(t)\right|} \geq C_{2} \frac{Q^{\prime}(t)}{|Q(t)|}, \quad \text { a.e. } \quad t \in I^{*} \backslash J^{*},
$$

then we write $W \in \mathcal{F}\left(C^{2}+\right)$.

Then we see that $w \in \mathcal{L}\left(C^{2}\right) \Leftrightarrow W \in \mathcal{F}\left(C^{2}\right)$ and $w \in \mathcal{L}\left(C^{2}+\right) \Leftrightarrow W \in \mathcal{F}\left(C^{2}+\right)$ where $W(t)=w(x), x=t^{2}$, from [6, Lemma 2.2]. In addition, we easily have the following:

Lemma 2.2. [1] Let $Q(t)=R(x), x=t^{2}$. Then we have

$$
w \in \mathcal{L}_{2} \Rightarrow W \in \mathcal{F}\left(C^{2}+\right),
$$

where $W(t)=w(x) ; x=t^{2}$.

On $\mathbb{R}$, we can consider the corresponding class to $\tilde{\mathcal{L}}_{v}$ as follows:

Definition 2.3. [11] Let $W=\exp (-Q) \in \mathcal{F}\left(C^{2}+\right)$ and $v \geq 2$ be an integer. Let $Q$ be a continuous and even function on $\mathbb{R}$. For the exponent $Q$, we assume the following:

(a) $Q^{(j)}(x)>0$, for $0 \leq j \leq v$ and $t \in \mathbb{R}^{+} /\{0\}$. 
(b) There exist positive constants $C_{i}>0$ such that for $i=1,2, \ldots, v-1$,

$$
Q^{(i+1)}(t) \leq C_{i} Q^{(i)}(t) \frac{Q^{\prime}(t)}{Q(t)}, \quad \text { a.e. } \quad x \in \mathbb{R}^{+} \backslash\{0\} .
$$

(c) There exist positive constants $C, c_{1}>0$, and $0 \leq \delta^{*}<1$ such that on $t \in\left(0, c_{1}\right)$,

$$
Q^{(v)}(t) \leq C\left(\frac{1}{t}\right)^{\delta^{*}}
$$

(d) There exists $c_{2}>0$ such that we have one among the following:

(d1) $T^{*}(t) / t$ is quasi-increasing on $\left(c_{2}, \infty\right)$,

(d2) $Q^{(v)}(t)$ is nondecreasing on $\left(c_{2}, \infty\right)$.

Then we write $W(t)=e^{-Q(t)} \in \tilde{\mathcal{F}}_{v}$.

Let $W \in \tilde{\mathcal{F}}_{v}$, and $v \geq 2$. For $\rho^{*}>-\frac{1}{2}$, we set

$$
W_{\rho *}(t):=|t|^{\rho *} W(t) .
$$

Then we can construct the orthonormal polynomials $P_{n, \rho *}(t)=P_{n}\left(W_{\rho *}^{2} ; t\right)$ of degree $n$ with respect to $W_{\rho^{*}}(t)$. That is,

$$
\int_{-\infty}^{\infty} P_{n, \rho *}(v) P_{m, \rho *}(v) W_{\rho *}^{2}(v) \mathrm{d} t=\delta_{n m}, \quad n, m=0,1,2, \ldots
$$

Let us denote the zeros of $P_{n, \rho^{*}}(t)$ by

$$
-\infty<t_{n n}<\cdots<t_{2 n}<t_{1 n}<\infty .
$$

There are many properties of $P_{n, \rho^{*}}(t)=P_{n}\left(W_{\rho^{*}} ; t\right)$ with respect to $W_{\rho^{*}}(t)$, $W \in \tilde{\mathcal{F}}_{\nu}, v=2,3, \ldots$ of Definition 2.3 in $[2,3,7,11-13]$. They were obtained by transformations from the results in [5,6]. Jung and Sakai [2, Theorem 3.3 and 3.6] estimate $P_{n, \rho *}^{(j)}\left(t_{k, n}\right), k=1,2, \ldots, n, j=1,2, \ldots, v$ and Jung and Sakai [1, Theorem 3.2 and 3.3] obtained analogous estimations with respect to $p_{n, \rho}^{(j)}\left(x_{k, n}\right), k=1,2, \ldots, n, j=1,2, \ldots, v$. In this article, we consider $w=\exp (-R) \in \tilde{\mathcal{L}}_{v}$ and $p_{n, \rho}(x)=p_{n}\left(w_{\rho} ; x\right)$. In the following, we give the transformation theorems.

Theorem 2.4. [13, Theorem 2.1] Let $W(t)=W(x)$ with $x=t^{2}$. Then the orthonormal polynomials $P_{n, \rho^{*}}(t)$ on $\mathbb{R}$ can be entirely reduced to the orthonormal polynomials $p_{n}$, $\rho$ $(x)$ in $\mathbb{R}^{+}$as follows: For $n=0,1,2, \ldots$,

$$
P_{2 n, 2 \rho+\frac{1}{2}}(t)=p_{n, \rho}(x) \text { and } P_{2 n+1,2 \rho-\frac{1}{2}}(t)=t p_{n, \rho}(x) .
$$

In this article, we will use the fact that $w_{\rho}(x)=x^{\rho} \exp (-R(x))$ is transformed into $W_{2 \rho}$ $+1 / 2(t)=|t|^{2 \rho+1 / 2} \exp (-Q(t))$ as meaning that

$$
\begin{aligned}
\int_{0}^{\infty} p_{n, \rho}(x) p_{m, \rho}(x) w_{\rho}^{2}(x) \mathrm{d} x & =2 \int_{0}^{\infty} p_{n, \rho}\left(t^{2}\right) p_{m, \rho}\left(t^{2}\right) t^{4 \rho+1} W^{2}(t) \mathrm{d} t \\
& =\int_{-\infty}^{\infty} P_{2 n, 2 \rho+1 / 2}(t) P_{2 m, 2 \rho+1 / 2}(t) W_{2 \rho+1 / 2}^{2}(t) \mathrm{d} t .
\end{aligned}
$$


Theorem 2.5. [1, Theorem 2.5] Let $Q(t)=R(x), x=t^{2}$. Then we have

$$
w(x)=\exp (-R(x)) \in \tilde{\mathcal{L}}_{v} \Rightarrow W(t)=\exp (-Q(t)) \in \tilde{\mathcal{F}}_{v} .
$$

In particular, we have

$$
Q^{(v)}(t) \leq C\left(\frac{1}{t}\right)^{\delta}
$$

where $0 \leq \delta<1$ is defined in (1.1).

For convenience, in the remainder of this article, we set as follows:

$$
\rho^{*}:=2 \rho+\frac{1}{2} \text { for } \rho>-\frac{1}{2}, \quad p_{n}(x):=p_{n, \rho}(x), \quad P_{n}(t):=P_{n, \rho^{*}}(t),
$$

and $x_{k, n}=x_{k, n, \rho}, t_{k n}=t_{k, n, \rho^{*}}$. Then we know that $\rho^{*}>-\frac{1}{2}$ and

$$
p_{n}(x)=P_{2 n, \rho^{*}}(t), x=t^{2}, \quad x_{k, n}=t_{k, 2 n^{\prime}}^{2} \quad t_{k, 2 n}>0, k=1,2, \ldots, n .
$$

In the following, we introduce useful notations:

(a) The Mhaskar-Rahmanov-Saff numbers $a_{v}$ and $a_{u}^{*}$ are defined as the positive roots of the following equations, that is,

$$
v=\frac{1}{\pi} \int_{0}^{1} a_{v} t R^{\prime}\left(a_{v} t\right)\{t(1-t)\}^{-\frac{1}{2}} d t, v>0
$$

and

$$
u=\frac{2}{\pi} \int_{0}^{1} a_{u}^{*} t Q^{\prime}\left(a_{u}^{*} t\right)\left(1-t^{2}\right)^{-\frac{1}{2}} d t, u>0
$$

(b) Let

$$
\eta_{n}=\left\{n T\left(a_{n}\right)\right\}^{-\frac{2}{3}} \quad \text { and } \quad \eta_{n}^{*}=\left\{n T^{*}\left(a_{n}^{*}\right)\right\}^{-\frac{2}{3}} .
$$

Then we have the following:

Lemma 2.6. [6, (2.5),(2.7),(2.9)]

$$
a_{n}=a_{2 n}^{*}, \quad \eta_{n}=4^{2 / 3} \eta_{2 n^{\prime}}^{*} \quad T\left(a_{n}\right)=\frac{1}{2} T^{*}\left(a_{2 n}^{*}\right) .
$$

To prove main results, we need some lemmas as follows:

Lemma 2.7. [13, Theorem 2.2, Lemma 3.7] For the minimum positive zero, $t_{[n / 2], n}([n / 2]$ is the largest integer $\leq n / 2$ ), we have

$$
t_{[n / 2], n} \sim a_{n}^{*} n^{-1}
$$

and for the maximum zero $t_{1 n}$ we have for large enough $n$,

$$
1-\frac{t_{1 n}}{a_{n}^{*}} \sim \eta_{n^{\prime}}^{*} \quad \eta_{n}^{*}=\left(n T^{*}\left(a_{n}^{*}\right)\right)^{-\frac{2}{3}}
$$


Moreover, for some constant $0<\varepsilon \leq 2$ we have

$$
T^{*}\left(a_{n}^{*}\right) \leq C n^{2-\varepsilon} .
$$

Remark 2.8. (a) Let $W(t) \in \mathcal{F}\left(C^{2}+\right)$. Then

(a-1) $T(x)$ is bounded $\Leftrightarrow T^{*}(t)$ is bounded.

(a-2) $T(x)$ is unbounded $\Rightarrow a_{n} \leq C(\eta) n^{\eta}$ for any $\eta>0$.

(a-3) $T\left(a_{n}\right) \leq C n^{2-\varepsilon}$ for some constant $0<\varepsilon \leq 2$.

(b) Let $w(x) \in \tilde{\mathcal{L}}_{v}$. Then

(b-1) $\rho>-1 / 2 \Rightarrow \rho^{*}>-1 / 2$.

(b-2) $1+2 \rho-\delta / 2 \geq 0$ for $\rho<-1 / 4 \Rightarrow 1+2 \rho^{*}-\delta^{*} \geq 0$ for $\rho^{*}<0$.

(b-3) $a_{n} \leq C n^{2 /(1+\nu-\delta)} \Rightarrow a_{n}^{*} \leq C n^{1 /\left(1+\nu-\delta^{*}\right)}$.

Proof of Remark 2.8. (a) (a-1) and (a-3) are easily proved from Lemma 2.6. From [11, Theorem 1.6], we know the following: When $T^{*}(t)$ is unbounded, for any $\eta>0$ there exists $C(\eta)>0$ such that

$$
a_{t}^{*} \leq C(\eta) t^{\eta}, \quad t \geq 1
$$

In addition, since $T(x)=T^{*}(t) / 2$ and $a_{n}=a_{2 n}^{*}$, we know that (a-2).

(b) Since $w(x) \in \tilde{\mathcal{L}}_{v}$, we know that $W(t) \in \tilde{\mathcal{F}}_{v}$ and $\delta^{*}=\delta$ by Theorem 2.5. Then from (2.3) and Lemma 2.6, we have (b-1), (b-2), and (b-3).

Lemma 2.9. [1, Lemma 3.6] For $j=1,2,3$,..., we have

$$
p_{n}^{(j)}(x)=\sum_{i=1}^{j}(-1)^{j-i} c_{j, i} P_{2 n}^{(i)}(t) t^{-2 j+i}
$$

where $c_{j, i}>0(1 \leq i \leq j, j=1,2, \ldots)$ satisfy the following relations: for $k=1,2, \ldots$,

$$
c_{k+1,1}=\frac{2 k-1}{2} c_{k, 1}, \quad c_{k+1, k+1}=\frac{1}{2^{k+1}}, \quad c_{1,1}=\frac{1}{2},
$$

and for $2 \leq i \leq k$,

$$
c_{k+1, i}=\frac{c_{k, i-1}+(2 k-i) c_{k, i}}{2} .
$$

\section{Proofs of main results}

Our main purpose is to obtain estimations of the coefficients $e_{s, i}(l, m, k, n), k=1,2, \ldots, 0$ $\leq s \leq l, s \leq i \leq m-1$.

Theorem 3.1. [1, Theorem 1.5] Let $w(x)=\exp (-R(x)) \in \mathcal{L}\left(C^{2}+\right)$ and let $\rho>-1 / 2$. For each $k=1,2, \ldots, n$ and $j=0,1, \ldots$, we have

$$
\left|p_{n, \rho}^{(j)}\left(x_{k, n}\right)\right| \leq C\left(\frac{n}{\sqrt{a_{2 n}-x_{k, n}}}\right)^{j-1} x_{k, n}^{-\frac{j-1}{2}}\left|p_{n, \rho}^{\prime}\left(x_{k, n}\right)\right| \text {. }
$$


Proof of Theorem 1.5. (a) From Theorem 3.1 we know that

$$
\left|l_{k, n}^{(j)}\left(x_{k, n}\right)\right|=\left|\frac{p_{n}^{(j+1)}\left(x_{k, n}\right)}{(j+1) p_{n}^{\prime}\left(x_{k, n}\right)}\right| \leq C\left(\frac{n}{\sqrt{a_{2 n}-x_{k, n}}}\right)^{j} x_{k, n}^{-\frac{j}{2}} .
$$

Then, assuming that (a) is true for $1 \leq m^{\prime}<m$, we have

$$
\begin{aligned}
\left|\left(l_{k, n}^{m}\right)^{(j)}\left(x_{k, n}\right)\right| & =\left|\sum_{s=0}^{j}\left(\begin{array}{l}
j \\
s
\end{array}\right)\left(l_{k, n}^{m-1}\right)^{(s)}\left(x_{k, n}\right) l_{k, n}{ }^{(j-s)}\left(x_{k, n}\right)\right| \\
& \leq C \sum_{s=0}^{j}\left(\frac{n}{\sqrt{a_{2 n}-x_{k, n}}}\right)^{s} x_{k, n}^{-\frac{s}{2}}\left(\frac{n}{\sqrt{a_{2 n}-x_{k, n}}}\right)^{j-s} x_{k, n}^{-\frac{j-s}{2}} \\
& \leq\left(\frac{n}{\sqrt{a_{2 n}-x_{k, n}}}\right)^{j} x_{k, n}^{-\frac{j}{2}} .
\end{aligned}
$$

Therefore, the result is proved by induction with respect to $m$.

(b) From (2) and (3), we know $e_{s, s}(l, m, k, n)=1 / s$ ! and the following recurrence relation: for $s+1 \leq i \leq m-1$,

$$
e_{s, i}(l, m, k, n)=-\sum_{p-s}^{i-1} \frac{1}{(i-p) !} e_{s, p}(l, m, k, n)\left(l_{k, n}\right)^{(i-p)}\left(x_{k, n}\right) .
$$

Therefore, we have the result by induction on $i$ and (3.5).

Theorem 3.2. [1, Theorem 1.6] Let $w(x)=\exp (-R(x)) \in \tilde{\mathcal{L}}_{v}$ and let $\rho>-1 / 2$. Suppose the same conditions as the assumptions of Theorem 1.6. For each $k=1,2, \ldots, n$ and $j=$ $1, \ldots, v$, we have

$$
\left|p_{n, \rho}^{(j)}\left(x_{k, n}\right)\right| \leq C\left(\frac{n}{\sqrt{a_{n}}-\sqrt{x_{k, n}}}+\frac{T\left(a_{n}\right)}{\sqrt{a_{n}}}\right)^{j-1} x_{k, n}^{-\frac{j-1}{2}}\left|p_{n, \rho}^{\prime}\left(x_{k, n}\right)\right|
$$

and in particular, if $j$ is even, then we have

$$
\begin{aligned}
\left|p_{n, \rho}^{(j)}\left(x_{k, n}\right)\right| \leq & C\left(\frac{T\left(a_{n}\right)}{\sqrt{a_{n} x_{k, n}}}+R^{\prime}\left(x_{k, n}\right)+\frac{1}{x_{k, n}}\right) \\
& \times\left(\frac{n}{\sqrt{a_{n}}-\sqrt{x_{k, n}}}+\frac{T\left(a_{n}\right)}{\sqrt{a_{n}}}\right)^{j-2} x_{k, n}^{-\frac{j-2}{2}}\left|p_{n, \rho}^{\prime}\left(x_{k, n}\right)\right| .
\end{aligned}
$$

Proof of Theorem 1.6. We use the induction method on $m$.

(a) For $m=1$, we have the result because of

$$
l_{k, n}^{(j)}\left(x_{k, n}\right)=\frac{p_{n}^{(j+1)}\left(x_{k, n}\right)}{(j+1) p_{n}^{\prime}\left(x_{k, n}\right)}, \quad j=1,2,3, \ldots
$$

and Theorem 3.2. Now we assume the theorem for $1 \leq m^{\prime}<m$. Then, we have the following: For $1 \leq 2 s-1 \leq v-1$, 


$$
\begin{aligned}
\left(l_{k, n}^{m}\right)^{(2 s-1)}\left(x_{k, n}\right)= & \sum_{r=0}^{s}\left(\begin{array}{c}
2 s-1 \\
2 r
\end{array}\right)\left(l_{k, n}^{m-1}\right)^{(2 r)}\left(x_{k, n}\right) l_{k, n}^{(2 s-2 r-1)}\left(x_{k, n}\right) \\
& +\sum_{r=0}^{s}\left(\begin{array}{c}
2 s-1 \\
2 r+1
\end{array}\right)\left(l_{k, n}^{m-1}\right)^{(2 r+1)}\left(x_{k, n}\right) l_{k, n}^{(2 s-2 r-2)}\left(x_{k, n}\right) .
\end{aligned}
$$

Since

$$
\frac{n}{\sqrt{a_{2 n}-x_{k, n}}} \leq \frac{n}{\sqrt{a_{2 n}}-\sqrt{x_{k, n}}}
$$

we have

$$
\begin{aligned}
& \left|\left(l_{k, n}^{m-1}\right)^{(2 r)}\left(x_{k, n}\right) l_{k, n}^{(2 s-2 r-1)}\left(x_{k, n}\right)\right| \\
& \leq C\left(\frac{T\left(a_{n}\right)}{\sqrt{a_{n} x_{k, n}}}+R^{\prime}\left(x_{k, n}\right)+\frac{1}{x_{k, n}}\right) \\
& \quad \times\left(\frac{n}{\sqrt{a_{2 n}-x_{k, n}}}\right)^{2 r}\left(\frac{n}{\sqrt{a_{2 n}}-\sqrt{x_{k, n}}}+\frac{T\left(a_{n}\right)}{\sqrt{a_{n}}}\right)^{2 s-2 r-2} x_{k, n}^{-s+1} \\
& \leq \\
& C\left(\frac{T\left(a_{n}\right)}{\sqrt{a_{n} x_{k, n}}}+R^{\prime}\left(x_{k, n}\right)+\frac{1}{x_{k, n}}\right) \\
& \quad \times\left(\frac{n}{\sqrt{a_{2 n}}-\sqrt{x_{k, n}}}+\frac{T\left(a_{n}\right)}{\sqrt{a_{n}}}\right)^{2 s-2} x_{k, n}^{-s+1},
\end{aligned}
$$

and similarly

$$
\begin{aligned}
\left|\left(l_{k, n}^{m-1}\right)^{(2 r+1)}\left(x_{k, n}\right) l_{k, n}^{(2 s-2 r-2)}\left(x_{k, n}\right)\right| \leq & C\left(\frac{T\left(a_{n}\right)}{\sqrt{a_{n} x_{k, n}}}+R^{\prime}\left(x_{k, n}\right)+\frac{1}{x_{k, n}}\right) \\
& \times\left(\frac{n}{\sqrt{a_{2 n}}-\sqrt{x_{k, n}}}+\frac{T\left(a_{n}\right)}{\sqrt{a_{n}}}\right)^{2 s-2} x_{k, n}^{-s+1} .
\end{aligned}
$$

Therefore, we have

$$
\begin{aligned}
\left|\left(l_{k, n}^{m}\right)^{(2 s-1)}\left(x_{k, n}\right)\right| \leq & C\left(\frac{T\left(a_{n}\right)}{\sqrt{a_{n} x_{k, n}}}+R^{\prime}\left(x_{k, n}\right)+\frac{1}{x_{k, n}}\right) \\
& \times\left(\frac{n}{\sqrt{a_{2 n}}-\sqrt{x_{k, n}}}+\frac{T\left(a_{n}\right)}{\sqrt{a_{n}}}\right)^{2 s-2} x_{k, n}^{-s+1} .
\end{aligned}
$$

(b) To prove the result, we proceed by induction on $i$. From (1.2) and (1.3) we know $e_{s, s}(l, m, k, n)=1 / s$ ! and the following recurrence relation: for $s+1 \leq i \leq m-1$,

$$
e_{s, i}(l, m, k, n)=-\sum_{p=s}^{i-1} \frac{1}{(i-p) !} e_{s, p}(l, m, k, n)\left(l_{k, n}^{m}\right)^{(i-p)}\left(x_{k, n}\right) .
$$

When $i-s$ is odd, we know that

$$
\left\{\begin{array}{l}
i-p: \text { odd, } \text { if } p-s: \text { even } \\
i-p: \text { even, } \text { if } p-s: \text { odd }
\end{array}\right.
$$


Then, we have (1.9) from (1.5), (1.8), (3.6), and the assumption of induction on $i$.

Theorem 3.3. [1, Theorem 1.7] Let $0<\varepsilon<1 / 4$. Let $\frac{1}{\varepsilon} \frac{a_{n}}{n^{2}} \leq x_{k, n} \leq \varepsilon a_{n}$ and let $s$ be a positive integer with $2 \leq 2 s \leq v-1$. Suppose the same conditions as the assumptions of Theorem 1.6. Then there exists $\beta(n, k), 0<D_{1} \leq \beta(n, k) \leq D_{2}$ for absolute constants $D_{1}$, $D_{2}$ such that the following equality holds:

$$
p_{n, \rho}^{(2 s+1)}\left(x_{k, n}\right)=(-1)^{s} \beta^{s}(2 n, k)\left(\frac{n}{\sqrt{a_{n}}}\right)^{2 s}\left(1+\rho_{s}\left(\varepsilon, x_{k, n}, n\right)\right) p_{n}^{\prime}\left(x_{k, n}\right) x_{k, n}^{-s}
$$

and $\left|\rho_{s}\left(\varepsilon, x_{k, n}, n\right)\right| \rightarrow 0$ as $n \rightarrow \infty$ and $\varepsilon \rightarrow 0$.

Lemma 3.4. [3, Theorem 2.5] Let $W \in \mathcal{F}\left(C^{2}+\right)$ and $r=1,2, \ldots$. Then, uniformly for $1 \leq k$ $\leq n$,

$$
\left|\frac{P_{n}^{(r)}\left(t_{k, n}\right)}{P^{\prime}{ }_{n}\left(t_{k, n}\right)}\right| \leq C\left(\frac{n}{\sqrt{a_{2 n}^{*}{ }^{2}-t_{k, n}^{2}}}\right)^{r-1} .
$$

Lemma 3.5. [2, Theorem 3.3] Let $\rho^{*}>-1 / 2$ and $W(x)=\exp (-Q(x)) \in \tilde{\mathcal{F}}_{v}, v \geq 2$. Assume that $1+2 \rho^{*}-\delta^{*} \geq 0$ for $\rho^{*}<0$ and if $T^{*}(t)$ is bounded, then assume

$$
a_{n}^{*} \leq C n^{1 /\left(1+\nu-\delta^{*}\right)},
$$

where $0 \leq \delta^{*}<1$ is defined in (2.1). Let $0<\alpha<1 / 2$. Let $\frac{1}{\varepsilon} \frac{a_{n}^{*}}{n} \leq\left|t_{k n}\right| \leq \varepsilon a_{n}^{*}$ and let $s$ be a positive integer with $2 \leq 2 s \leq v$. Then there exists $\mu(\varepsilon, n)>0$ such that

$$
\left|P_{n}^{(2 s)}\left(t_{k, n}\right)\right| \leq C \mu(\varepsilon, n)\left(\frac{n}{a_{n}}\right)^{2 s-1}\left|P_{n}^{\prime}\left(t_{k, n}\right)\right|
$$

and $\mu(\varepsilon, n) \rightarrow 0$ as $n \rightarrow \infty$ and $\varepsilon \rightarrow 0$.

Proof of Theorem 1.7. By Lemma 2.9, we have

$$
\begin{aligned}
\left|p_{n}^{(2 s)}\left(x_{k, n}\right)\right| & =\left|\sum_{i=1}^{2 s}(-1)^{2 s-i} c_{2 s, i} P_{2 n}^{(i)}\left(t_{k, n}\right) t_{k, n}^{-4 s+i}\right| \\
& \leq C\left|c_{2 s, 2 s} P_{2 n}^{(2 s)}\left(t_{k, n}\right) t_{k, n}^{-2 s}\right|+\left|\sum_{i=1}^{2 s-1}(-1)^{2 s-i} c_{2 s, i} P_{2 n}^{(i)}\left(t_{k, n}\right) t_{k, n}^{-4 s+i}\right| .
\end{aligned}
$$

Since, we have by Lemma 3.5,

$$
\left|c_{2 s, 2 s} P_{2 n}^{(2 s)}\left(t_{k, n}\right) t_{k, n}^{-2 s}\right| \leq C \mu(\varepsilon, 2 n)\left(\frac{n}{a_{2 n}^{*}}\right)^{2 s-1}\left|P_{2 n}^{\prime}\left(t_{k, n}\right)\right|\left|t_{k, n}\right|^{-2 s}
$$

and by Lemma 3.4,

$$
\begin{aligned}
& \left|\sum_{i=1}^{2 s-1}(-1)^{2 s-i} c_{2 s, i} P_{2 n}^{(i)}\left(t_{k, n}\right) t_{k, n}^{-4 s+i}\right| \\
\leq & C\left(\frac{n}{a_{2 n}^{*}}\right)^{2 s-1}\left|P_{2 n}^{\prime}\left(t_{k, n}\right)\right|\left|t_{k, n}\right|^{-2 s} \sum_{i=1}^{2 s-1}\left(\frac{n}{a_{2 n}^{*}}\left|t_{k, n}\right|\right)^{-2 s+i} \\
\leq & C \varepsilon\left(\frac{n}{a_{2 n}^{*}}\right)^{2 s-1}\left|P_{2 n}^{\prime}\left(t_{k, n}\right)\right|\left|t_{k, n}\right|^{-2 s},
\end{aligned}
$$


we have

$$
\begin{aligned}
\left|p_{n}^{(2 s)}\left(x_{k, n}\right)\right| & \leq C \delta(\varepsilon, n)\left(\frac{n}{a_{2 n}^{*}}\right)^{2 s-1}\left|P_{2 n}^{\prime}\left(t_{k, n}\right)\right|\left|t_{k, n}\right|^{-2 s} \\
& \leq C \delta(\varepsilon, n)\left(\frac{n}{\sqrt{a_{n}}}\right)^{2 s-1}\left|p_{n}^{\prime}\left(x_{k, n}\right)\right| x_{k, n}^{-\frac{(2 s-1)}{2}},
\end{aligned}
$$

where $\delta(\varepsilon, n)=\mu(\varepsilon, 2 n)+\varepsilon . \quad \square$

Here we can estimate the coefficients $e_{i}(v, k, n)$ of the fundamental polynomials $h_{k n}(v ; x)$.

For $j=0,1, \ldots$, define $\varphi_{j}(1):=(2 j+1)^{-1}$ and for $k \geq 2$,

$$
\varphi_{j}(k):=\sum_{r=0}^{j} \frac{1}{2 j-2 r+1}\left(\begin{array}{c}
2 j \\
2 r
\end{array}\right) \varphi_{r}(k-1) .
$$

Proof of Theorem 1.8. In a manner analogous to the proof of Theorem 1.6 (a), we use mathematical induction with respect to $m$.

(a) From Theorem 1.7, we know that for $1 \leq 2 s-1 \leq v-1$,

$$
\left|l_{k, n}^{(2 s-1)}\left(x_{k, n}\right)\right|=\left|\frac{p_{n}^{(2 s)}\left(x_{k, n}\right)}{2 s p_{n}^{\prime}\left(x_{k, n}\right)}\right| \leq C \delta(\varepsilon, n)\left(\frac{n}{\sqrt{a_{n}}}\right)^{2 s-1} x_{k, n}^{-\frac{2 s-1}{2}} .
$$

From Theorem 1.5, we know that for $x_{k, n} \leq a_{n} / 4$,

$$
\left|\left(l_{k, n}^{m}\right)^{(j)}\left(x_{k, n}\right)\right| \leq C\left(\frac{n}{\sqrt{a_{n}}}\right)^{j} x_{k, n}^{-\frac{j}{2}} .
$$

Then, we have by mathematical induction on $m$,

$$
\begin{aligned}
\left|\left(l_{k, n}^{m}\right)^{(2 s-1)}\left(x_{k, n}\right)\right| \leq & C \sum_{r=0}^{s}\left(\begin{array}{c}
2 s-1 \\
2 r
\end{array}\right)\left|\left(l_{k, n}^{m-1}\right)^{(2 r)}\left(x_{k, n}\right) l_{k, n}^{(2 s-2 r-1)}\left(x_{k, n}\right)\right| \\
& +\sum_{r=0}^{s}\left(\begin{array}{c}
2 s-1 \\
2 r+1
\end{array}\right)\left|\left(l_{k, n}^{m-1}\right)^{(2 r+1)}\left(x_{k, n}\right) l_{k, n}^{(2 s-2 r-2)}\left(x_{k, n}\right)\right| \\
\leq & C \delta(\varepsilon, n)\left(\frac{n}{\sqrt{a_{n}}}\right)^{2 s-1} x_{k, n}^{-\frac{2 s-1}{2}} .
\end{aligned}
$$

(b) From Theorem 3.3, we know that for $0 \leq 2 s \leq v-1$,

$$
\begin{aligned}
l_{k, n}^{(2 s)}\left(x_{k, n}\right) & =\frac{p_{n}^{(2 s+1)}\left(x_{k, n}\right)}{(2 s+1) p_{n}^{\prime}\left(x_{k, n}\right)} \\
& =(-1)^{s} \phi_{s}(1) \beta^{s}(2 n, k)\left(\frac{n}{\sqrt{a_{n}}}\right)^{2 s} x_{k, n}^{-s}\left(1+\rho_{s}\left(\varepsilon, x_{k, n}, n\right)\right) .
\end{aligned}
$$

If we let $\xi_{s}\left(1, \varepsilon, x_{k, n}, n\right)=\rho_{s}\left(\varepsilon, x_{k, n}, n\right)$, then (1.11) holds for $m=1$ because $\mid \xi_{s}\left(1, \varepsilon, x_{k, n}\right.$ $n) \mid \rightarrow 0$ as $n \rightarrow \infty$ and $\varepsilon \rightarrow 0$. Now, we split $\left(l_{k, n}^{m}\right)^{(2 s)}\left(x_{k, n}\right)$ into two terms as follows:

$$
\begin{aligned}
\left(l_{k, n}^{m}\right)^{(2 s)}\left(x_{k, n}\right)= & \sum_{0 \leq 2 r \leq 2 s}\left(\begin{array}{c}
2 s \\
2 r
\end{array}\right)\left(l_{k, n}^{m-1}\right)^{(2 r)}\left(x_{k, n}\right) l_{k, n}^{(2 s-2 r)}\left(x_{k, n}\right) \\
& +\sum_{1 \leq 2 r-1 \leq 2 s}\left(\begin{array}{c}
2 s \\
2 r-1
\end{array}\right)\left(l_{k, n}^{m-1}\right)^{(2 r-1)}\left(x_{k, n}\right) l_{k, n}^{(2 s-2 r+1)}\left(x_{k, n}\right) .
\end{aligned}
$$


For the second term, we have from (1.10),

$$
\left|\sum_{1 \leq 2 r-1 \leq 2 s}\left(\begin{array}{c}
2 s \\
2 r-1
\end{array}\right)\left(l_{k, n}^{m-1}\right)^{(2 r-1)}\left(x_{k, n}\right) l_{k, n}^{(2 s-2 r+1)}\left(x_{k, n}\right)\right| \leq C \delta^{2}(\varepsilon, n)\left(\frac{n}{\sqrt{a_{n}}}\right)^{2 s} x_{k, n}^{-(2, n .11)}
$$

For the first term, we let $\xi_{s}(m)=\xi_{s}\left(m, \varepsilon, x_{k, n}, n\right)$ for convenience. Then we know that

$$
l_{k, n}^{(2 s-2 r)}\left(x_{k, n}\right)=(-1)^{s-r} \phi_{s-r}(1) \beta^{s-r}(2 n, k)\left(\frac{n}{\sqrt{a_{n}}}\right)^{2 s-r} x_{k, n}^{-(s-r)}\left(1+\xi_{s-r}(1)\right)
$$

and $\left|\xi_{s-r}(1)\right| \rightarrow 0$ as $n \rightarrow \infty$ and $\varepsilon \rightarrow 0$. By mathematical induction, we assume for 0 $\leq 2 r \leq 2 s$

$$
\left(l_{k, n}^{m-1}\right)^{(2 r)}\left(x_{k, n}\right)=(-1)^{r} \phi_{r}(m-1) \beta^{r}(2 n, k)\left(\frac{n}{\sqrt{a_{n}}}\right)^{2 r} x_{k, n}^{-r}\left(1+\xi_{r}(m-1)\right)
$$

and $\left|\xi_{r}(m-1)\right| \rightarrow 0$ as $n \rightarrow \infty$ and $\varepsilon \rightarrow 0$. Then, since

$$
\begin{array}{r}
\left(l_{k, n}^{m-1}\right)^{(2 r)}\left(x_{k, n}\right) l_{k, n}^{(2 s-2 r)}\left(x_{k, n}\right)=(-1)^{s} \beta^{s}(2 n, k)\left(\frac{n}{\sqrt{a_{n}}}\right)^{2 s} x_{k, n}^{-s} \\
\times \phi_{r}(m-1) \phi_{s-r}(1)\left(1+\xi_{r}(m-1)\right)\left(1+\xi_{s-r}(1)\right),
\end{array}
$$

we have for $0 \leq 2 r \leq 2 s$, using the definition of (3.7),

$$
\begin{aligned}
& \sum_{0 \leq 2 r \leq 2 s}\left(\begin{array}{c}
2 s \\
2 r
\end{array}\right)\left(l_{k, n}^{m-1}\right)^{(2 r)}\left(x_{k, n}\right) l_{k, n}^{(2 s-2 r)}\left(x_{k, n}\right) \\
= & (-1)^{s} \beta^{s}(2 n, k)\left(\frac{n}{\sqrt{a_{n}}}\right)^{2 s} x_{k, n}^{-s} \\
& \times \sum_{0 \leq 2 r \leq 2 s}\left(\begin{array}{c}
2 s \\
2 r
\end{array}\right) \phi_{r}(m-1) \phi_{s-r}(1)\left(1+\xi_{r}(m-1)\right)\left(1+\xi_{s-r}(1)\right) \\
= & (-1)^{s} \phi_{s}(m) \beta^{s}(2 n, k)\left(\frac{n}{\sqrt{a_{n}}}\right)^{2 s} x_{k, n}^{-s}+(-1)^{s} \beta^{s}(2 n, k)\left(\frac{n}{\sqrt{a_{n}}}\right)^{2 s} x_{k, n}^{-s} \\
& \times \sum_{0 \leq 2 r \leq 2 s}\left(\begin{array}{c}
2 s \\
2 r
\end{array}\right) \phi_{r}(m-1) \phi_{s-r}(1)\left(\xi_{r}(m-1)+\xi_{s-r}(1)+\xi_{r}(m-1) \xi_{s-r}(1)\right) .
\end{aligned}
$$

Here, we consider (3.10). If we let

$$
\begin{aligned}
& \xi_{s}\left(m, \varepsilon, x_{k, n}, n\right)=\xi_{s}(m)= \\
& \sum_{0 \leq 2 r \leq 2 s}\left(\begin{array}{c}
2 s \\
2 r
\end{array}\right) \frac{\phi_{r}(m-1) \phi_{s-r}(1)}{\phi_{s}(m)}\left(\xi_{r}(m-1)+\xi_{s-r}(1)+\xi_{r}(m-1) \xi_{s-r}(1)\right) \\
& \quad+\sum_{1 \leq 2 r-1 \leq 2 s}\left(\begin{array}{c}
2 s \\
2 r-1
\end{array}\right) \frac{\left(l_{k, n}^{m-1}\right)^{(2 r-1)}\left(x_{k, n}\right) l_{k, n}^{(2 s-2 r+1)}\left(x_{k, n}\right)}{(-1)^{s} \phi_{s}(m) \beta^{s}(2 n, k)\left(\frac{n}{\sqrt{a_{n}}}\right)^{2 s} x_{k, n}^{-s}}
\end{aligned}
$$


then we have

$$
\begin{aligned}
\xi_{s}(m) \leq & \sum_{0 \leq 2 r \leq 2 s}\left(\begin{array}{l}
2 s \\
2 r
\end{array}\right) \frac{\phi_{r}(m-1) \phi_{s-r}(1)}{\phi_{s}(m)}\left(\xi_{r}(m-1)+\xi_{s-r}(1)+\xi_{r}(m-1) \xi_{s-r}(1)\right) \\
& +C \frac{\delta^{2}(\varepsilon, n)\left(\frac{n}{\sqrt{a_{n}}}\right)^{2 s} x_{k, n}^{-s}}{(-1)^{s} \phi_{s}(m) \beta^{s}(2 n, k)\left(\frac{n}{\sqrt{a_{n}}}\right)^{2 s} x_{k, n}^{-s}} \text { by the definition of }(3.11) \\
\leq & \sum_{0 \leq 2 r \leq 2 s}\left(\begin{array}{l}
2 s \\
2 r
\end{array}\right) \frac{\phi_{r}(m-1) \phi_{s-r}(1)}{\phi_{s}(m)}\left(\xi_{r}(m-1)+\xi_{s-r}(1)+\xi_{r}(m-1) \xi_{s-r}(1)\right) \\
& +C^{\prime} \delta^{2}(\varepsilon, n) .
\end{aligned}
$$

Then, we know that (1.11) holds and $\left|\xi_{i}(j)\right| \rightarrow 0$ as $n \rightarrow \infty$ and $\varepsilon \rightarrow 0$, using mathematical induction on $m$. Therefore, we have the result.

We rewrite the relation (3.7) in the form for $v=1,2,3 \ldots$,

$$
\phi_{0}(v):=1
$$

and for $j=1,2,3 \ldots, v=2,3,4, \ldots$,

$$
\phi_{j}(v)-\phi_{j}(v-1)=\frac{1}{2 j+1} \sum_{r=0}^{j-1}\left(\begin{array}{c}
2 j+1 \\
2 r
\end{array}\right) \phi_{r}(v-1) .
$$

Now, for every $j$ we will introduce an auxiliary polynomial determined by $\left\{\Psi_{j}(\gamma)\right\}_{j=1}^{\infty}$ as the following lemma:

Lemma 3.6. [4, Lemma 11] (i) For $j=0,1,2$..., there exists a unique polynomial $\Psi_{j}$ (y) of degree $j$ such that

$$
\Psi_{j}(v)=\phi_{j}(v), \quad v=1,2,3, \ldots
$$

(ii) $\Psi_{0}(y)=1$ and $\Psi_{j}(0)=0, j=1,2, \ldots$

Since $\Psi_{j}(y)$ is a polynomial of degree $j$, we can replace $\varphi_{j}(v)$ in (3.7) with $\Psi_{j}(y)$, that is,

$$
\Psi_{j}(\gamma)=\sum_{r=0}^{j} \frac{1}{2 j-2 r+1}\left(\begin{array}{c}
2 j \\
2 r
\end{array}\right) \Psi_{r}(\gamma-1), \quad j=0,1,2, \ldots
$$

for an arbitrary $y$ and $j=0,1,2, \ldots$. We use the notation $F_{k n}(x, y)=\left(l_{k, n}(x)\right)^{y}$ which coincides with $l_{k, n}^{\gamma}(x)$ if $y$ is an integer. Since $l_{k, n}\left(x_{k, n}\right)=1$, we have $F_{k n}(x, t)>0$ for $x$ in a neighborhood of $x_{k, n}$ and an arbitrary real number $t$.

We can show that $(\partial / \partial x)^{j} F_{k n}\left(x_{k, n}, y\right)$ is a polynomial of degree at most $j$ with respect to $y$ for $j=0,1,2, \ldots$, where $(\partial / \partial x)^{j} F_{k n}\left(x_{k, n}, y\right)$ is the $j$ th partial derivative of $F_{k n}(x, y)$ with respect to $x$ at $\left(x_{k, n}, y\right)$ [14, p. 199]. We prove these facts by induction on $j$. For $j=0$ it is trivial. Suppose that it holds for $j \geq 0$. To simplify the notation, let $F(x)=F_{k n}(x, y)$ and $l(x)=l_{k, n}(x)$ for a fixed $y$. Then $F^{\prime}(x) l(x)=y l^{\prime}(x) F(x)$. By Leibniz's rule, we easily see that 


$$
F^{(j+1)}\left(x_{k, n}\right)=-\sum_{s=0}^{j-1}\left(\begin{array}{l}
j \\
s
\end{array}\right) F^{(s+1)}\left(x_{k, n}\right) l^{(j-s)}\left(x_{k, n}\right)+\gamma \sum_{s=0}^{j}\left(\begin{array}{l}
j \\
s
\end{array}\right) l^{(s+1)}\left(x_{k, n}\right) F^{(j-s)}\left(x_{k, n}\right),
$$

which shows that $F^{(j+1)}\left(x_{k, n}\right)$ is a polynomial of degree at most $j+1$ with respect to $y$. Let $P_{k n}^{[j]}(y), j=0,1,2, \ldots$ be defined by

$$
(\partial / \partial x)^{2 j} F_{k n}\left(x_{k, n}, y\right)=(-1)^{j} \beta^{j}(2 n, k)\left(\frac{n}{\sqrt{a_{n}}}\right)^{2 j} x_{k, n}^{-j} \Psi_{j}(\gamma)+P_{k n}^{[j]}(\gamma) .
$$

Then $P_{k n}^{[j]}(y)$ is a polynomial of degree at most $2 j$.

By Theorem 1.8 (1.11), we have the following:

Lemma 3.7. [4, Lemma 12] Let $j=0,1,2, \ldots$, and $M$ be a positive constant. Let $0<\varepsilon<$ $1 / 4, \frac{1}{\varepsilon} \frac{a_{n}}{n^{2}} \leq x_{k, n} \leq \varepsilon a_{n}$, and $|y| \leq M$. Then

(a) there exists $\kappa_{j}\left(\varepsilon, x_{k, n}, n\right)>0$ such that

$$
\left|(\partial / \partial y)^{s} P_{k n}^{[j]}(y)\right| \leq C \kappa_{j}\left(\varepsilon, x_{k, n}, n\right)\left(\frac{n}{\sqrt{a_{n}}}\right)^{2 j} x_{k, n^{\prime}}^{-j} \quad s=0,1
$$

and $\kappa_{j}\left(\varepsilon, x_{k}, n, n\right) \rightarrow 0$ as $n \rightarrow \infty$ and $\varepsilon \rightarrow 0$.

(b) there exists $\gamma_{j}(\varepsilon, n)>0$ such that

$$
\left|(\partial / \partial x)^{2 j+1} F_{k n}\left(x_{k, n}, y\right)\right| \leq C \gamma_{j}(\varepsilon, n)\left(\frac{n}{\sqrt{a_{n}}}\right)^{2 j+1} x_{k, n}^{-\frac{2 j+1}{2}}
$$

and $\gamma_{j}(\varepsilon, n) \rightarrow 0$ as $n \rightarrow \infty$ and $\varepsilon \rightarrow 0$.

Lemma 3.8. [4, Lemma 13] If $y<0$, then for $j=0,1,2 \ldots$,

$$
(-1)^{j} \Psi_{j}(y)>0
$$

Lemma 3.9. For positive integers $s$ and $m$ with $1 \leq m \leq v$,

$$
\sum_{r=0}^{s}\left(\begin{array}{l}
2 s \\
2 r
\end{array}\right) \Psi_{r}(-m) \varphi_{s-r}(m)=0
$$

Proof. If we let $C_{s}(\gamma)=\sum_{r=0}^{s}\left(\begin{array}{l}2 s \\ 2 r\end{array}\right) \Psi_{r}(-\gamma) \Psi_{s-r}(\gamma)$, then it suffices to show that $C_{s}$ $(m)=0$. For every $s$,

$$
\begin{aligned}
0= & \left(l_{k, n}^{-m+m}\right)^{2 s}\left(x_{k, n}\right)=\sum_{i=0}^{2 s}\left(\begin{array}{c}
2 s \\
i
\end{array}\right)\left(l_{k, n}^{-m}\right)^{(i)}\left(x_{k, n}\right)\left(l_{k, n}^{m}\right)^{(2 s-i)}\left(x_{k, n}\right) \\
= & \sum_{r=0}^{s}\left(\begin{array}{c}
2 s \\
2 r
\end{array}\right)(\partial / \partial x)^{2 r} F_{k n}\left(x_{k, n},-m\right)\left(l_{k, n}^{m}\right)^{(2 s-2 r)}\left(x_{k, n}\right) \\
& +\sum_{r=0}^{s-1}\left(\begin{array}{c}
2 s \\
2 r+1
\end{array}\right)(\partial / \partial x)^{2 r+1} F_{k n}\left(x_{k, n},-m\right)\left(l_{k, n}^{m}\right)^{(2 s-2 r-1)}\left(x_{k, n}\right) .
\end{aligned}
$$


By (1.11), (3.12) and (3.13), we see that the first sum $\sum_{r=0}^{s}$ has the form of

$$
\sum_{r=0}^{s}=(-1)^{s} \beta^{s}(2 n, k)\left(\frac{n}{\sqrt{a_{n}}}\right)^{2 s} x_{k, n}^{-s}\left(\sum_{r=0}^{s}\left(\begin{array}{c}
2 s \\
2 r
\end{array}\right) \Psi_{r}(-m) \phi_{s-r}(m)+\tilde{\eta}_{s}\left(-m, \varepsilon, x_{k, n}, n\right)\right) .
$$

Then, since

$$
\begin{aligned}
\tilde{\eta}_{s}\left(-m, \varepsilon, x_{k, n}, n\right)= & \sum_{r=0}^{s}\left(\begin{array}{l}
2 s \\
2 r
\end{array}\right) \Psi_{r}(-m) \phi_{s-r}(m) \xi_{s-r}\left(m, \varepsilon, x_{k, n}, n\right) \\
& +\sum_{r=0}^{s}\left(\begin{array}{l}
2 s \\
2 r
\end{array}\right)(-1)^{-r} \beta^{-r}(2 n, k)\left(\frac{n}{\sqrt{a_{n}}}\right)^{-2 r} x_{k, n}^{r} \\
& \times \phi_{s-r}(m) P_{k n}^{[r]}(-m)\left(1+\xi_{s-r}\left(m, \varepsilon, x_{k, n}, n\right)\right),
\end{aligned}
$$

we know that $\left|\tilde{\eta}_{s}\left(-m, \varepsilon, x_{k, n}, n\right)\right| \rightarrow 0$ as $n \rightarrow \infty$ and $\varepsilon \rightarrow 0$ (see (3.12)). By (3.14) and (3.8), the second sum $\sum_{r=0}^{s-1}$ is bounded by $C\left(\frac{n}{\sqrt{a_{n}}}\right)^{2 s+1} x_{k, n}^{-\frac{2 s+1}{2}} \sum_{r=0}^{2 s-1} \gamma_{r}(\varepsilon, n)$, and we know that $\sum_{r=0}^{2 s-1} \gamma_{r}(\varepsilon, n) \rightarrow 0$ as $n \rightarrow \infty$ and $\varepsilon \rightarrow 0$. Therefore, we obtain the following result: for every $s$,

$$
0=\sum_{r=0}^{s}\left(\begin{array}{c}
2 s \\
2 r
\end{array}\right) \Psi_{r}(-m) \Psi_{s-r}(m) .
$$

Theorem 1.9 is important to show a divergence theorem with respect to $L_{n}(m, f ; x)$, where $m$ is an odd integer.

Proof of Theorem 1.9. We prove (1.12) by induction on $s$. Since $e_{0}(m, k, n)=1$ and $\Psi_{0}(y)=1,(1.12)$ holds for $s=0$. From (3.6) we write $e_{2 s}(m, k, n)$ in the form of

$$
\begin{aligned}
e_{2 s}(m, k, n)= & -\sum_{r=0}^{s-1} \frac{1}{(2 s-2 r) !} e_{2 r}(m, k, n)\left(l_{k, n}^{m}\right)^{(2 s-2 r)}\left(x_{k, n}\right) \\
& \quad-\sum_{r=1}^{s} \frac{1}{(2 s-2 r+1) !} e_{2 r-1}(m, k, n)\left(l_{k, n}^{m}\right)^{(2 s-2 r+1)}\left(x_{k, n}\right) \\
= & I+I I .
\end{aligned}
$$

From (1.6), we know that for $x_{k, n} \leq a_{n} / 4$,

$$
\left|e_{j}(l, m, k, n)\right| \leq C\left(\frac{n}{\sqrt{a_{n}}}\right)^{j} x_{k, n}^{-\frac{j}{2}}
$$

Then, by (1.10) and (3.15), $|I I|$ is bounded by $C \sum_{r=1}^{s} \delta(\varepsilon, n)\left(\frac{n}{\sqrt{a_{n}}}\right)^{2 s} x_{k, n}^{-s}$. For $0 \leq i<s$, we suppose (1.12). Then, we have for $I$,

$$
\begin{aligned}
-\sum_{r=0}^{s-1}=\frac{(-1)^{s+1}}{(2 s) !} \beta^{s}(2 n, k)\left(\frac{n}{\sqrt{a_{n}}}\right)^{2 s} x_{k, n}^{-s} \\
\quad \times \sum_{r=0}^{s-1}\left(\begin{array}{c}
2 s \\
2 r
\end{array}\right) \Psi_{r}(-m) \phi_{s-r}(m)\left(1+\eta_{r}\right)\left(1+\xi_{s-r}\right),
\end{aligned}
$$


where $\xi_{s-r}:=\xi_{s-r}\left(m, \varepsilon, x_{k, n}, n\right)$ and $\eta_{r}:=\eta_{r}\left(m, \varepsilon, x_{k, n}, n\right)$ which are defined in (1.11) and (1.12). Then, using Lemma 3.9 and $\varphi_{0}(m)=1$, we have the following form:

$$
e_{2 s}(m, k, n)=\frac{(-1)^{s}}{(2 s) !} \Psi_{s}(-m) \beta^{s}(2 n, k)\left(\frac{n}{\sqrt{a_{n}}}\right)^{2 s} x_{k, n}^{-s}\left(1+\eta_{s}\left(m, \varepsilon, x_{k, n}, n\right)\right) .
$$

Here, since

$$
\begin{aligned}
\eta_{s}\left(m, \varepsilon, x_{k, n}, n\right)= & \sum_{r=0}^{s-1}\left(\begin{array}{c}
2 s \\
2 r
\end{array}\right) \Psi_{r}(-m) \phi_{s-r}(m)\left(\eta_{r}+\xi_{s-r}+\eta_{r} \xi_{s-r}\right) \\
& +(-1)^{s} \beta^{-s}(2 n, k)\left(\frac{n}{\sqrt{a_{n}}}\right)^{-2 s} x_{k, n}^{s} \frac{(2 s) !}{\Psi_{s}(-m)} I I
\end{aligned}
$$

we see that $\left|\eta_{s}\left(m, \varepsilon, x_{k, n}, n\right)\right| \rightarrow 0$ as $n \rightarrow \infty$ and $\varepsilon \rightarrow 0$ (recall above estimation of $|I I|)$. Therefore, we proved the result.

Lemma 3.10. [5, Theorem 1.3] Let $\rho>-\frac{1}{2}$ and $w(x)\left(C^{2}+\right)$. There exists $n_{0}$ such that uniformly for $n \geq n_{0}$, we have the following:

(a) For $1 \leq j \leq n$,

$$
\left|p_{n, \rho}^{\prime}\left(x_{j, n}\right)\right| w_{\rho}\left(x_{j, n}\right) \tilde{\varphi}_{n}\left(x_{j, n}\right)^{-1}\left[x_{j, n}\left(a_{n}-x_{j, n}\right)\right]^{-1 / 4}
$$

(b) For $j \leq n-1$ and $x \in\left[x_{j+1, n}, x_{j, n}\right]$,

$$
\begin{aligned}
& \left|p_{n, \rho}(x)\right| w(x)\left(x+\frac{a_{n}}{n^{2}}\right)^{\rho} \\
& \sim \min \left\{\left|x-x_{j, n}\right|,\left|x-x_{j+1, n}\right|\right\} \varphi_{n}\left(x_{j, n}\right)^{-1}\left[x_{j, n}\left(a_{n}-x_{j, n}\right)\right]^{-1 / 4} .
\end{aligned}
$$

(c) For $0<a \leq x_{k, n} \leq b<\infty$,

$$
\left|p_{n, \rho}^{\prime}\left(x_{k, n}\right)\right| w_{\rho}\left(x_{k, n}\right) \sim \frac{n}{a_{n}^{3 / 4}}
$$

(d) For $0<a \leq x_{k+1, n}, x_{k, n} \leq b<\infty$ and $\left.x \in\left[\left(x_{k+1, n}+x_{k, n}\right) / 2 x_{k, n}+x_{k-1, n}\right) / 2\right]$,

$$
\left|p_{n, \rho}(x)\right| w(x)\left(x+\frac{a_{n}}{n^{2}}\right)^{\rho} \sim \frac{1}{a_{n}^{1 / 4}} .
$$

Moreover, for $0<a \leq x \leq b<\infty$, there exists a constant $C>0$ such that

$$
\left|p_{n, \rho}(x)\right| w(x)\left(x+\frac{a_{n}}{n^{2}}\right)^{\rho} \leq C \frac{1}{a_{n}^{1 / 4}}
$$

(e) Uniformly for $n \geq 1$ and $1 \leq j<n$,

$$
x_{j, n}-x_{j+1, n} \sim \varphi_{n}\left(x_{j, n}\right) .
$$


(f) Let $\Lambda$ be defined in Definition 1.2 (d). There exists $C>0$ such that for $n \geq 1$,

$$
a_{n} \leq C n^{1 / \Lambda} .
$$

Proof. (a) and (b) follow from [5, Theorem 1.3]. (e) follows from [5, Theorem 1.4]. We need to prove (c), (d), and (f).

(c) For $0<a \leq x_{k, n} \leq b<\infty$, we have (2.11);

$$
\varphi_{n}\left(x_{k, n}\right) \sim \frac{\sqrt{a_{n}}}{n},
$$

so applying (a), we have the result.

(d) Let $0<a \leq x_{k+1, n}<x_{k, n} \leq b<\infty$. We take a constant $\delta>0$ as

$$
\min \left\{\left|x-x_{k, n}\right|,\left|x-x_{k+1, n}\right|\right\}=\delta \frac{\sqrt{a_{n}}}{n} .
$$

Then, by (b) we have

$$
\left|p_{n, \rho}(x)\right| w(x)\left(x+\frac{a_{n}}{n^{2}}\right)^{\rho} \sim \delta \frac{\sqrt{a_{n}}}{n} \frac{n}{a_{n}^{3 / 4}}=\delta \frac{1}{a_{n}^{1 / 4}} .
$$

Moreover, by [6, Theorem 1.2] the second inequality holds.

(f) We see

$$
\frac{R^{\prime}(x)}{R(x)}=\frac{T(x)}{x} \geq \frac{\Lambda}{x}
$$

so that by an integration, $R(x) \geq R(1) x^{\Lambda}$ for $x \geq 1$, and hence we have

$$
R^{\prime}(x) \geq \Lambda R(1) x^{\Lambda-1} \quad(x \geq 1) .
$$

Since $\lim _{n \rightarrow \infty} a_{n}=\infty$, we can choose $n_{0}$ such that $a_{n} \geq 2$ for all $n \geq n_{0}$. Then for some $C_{1}>0$,

$$
\begin{aligned}
& n=\frac{2}{\pi} \int_{0}^{1} \frac{a_{n} t R^{\prime}\left(a_{n} t\right)}{\sqrt{t(1-t)}} \mathrm{d} t \geq \frac{2}{\pi} \int_{0}^{1} \frac{a_{n} t \Lambda R(1)\left(a_{n} t\right)^{\Lambda-1}}{\sqrt{t(1-t)}} \mathrm{d} t \\
& \geq a_{n}^{\Lambda} \frac{2 \Lambda R(1)}{\pi} \int_{1 / 2}^{1} \frac{t^{\Lambda}}{\sqrt{t(1-t)}} \mathrm{d} t=: C_{1}^{\Lambda} a_{n}^{\Lambda} .
\end{aligned}
$$

Hence, we have the result.

Lemma 3.11. Let the function $h_{k n}(m ; x)$ be defined by (1.4) and let $0<c<a<b<d<$ $\infty$. Then we have

$$
\max _{a \leq x \leq b} \sum_{c \leq x_{k, n} \leq d}\left|l_{k, n}^{m}(x) \sum_{i=0}^{m-2} e_{i}(m, k, n)\left(x-x_{k, n}\right)^{i}\right| \leq C .
$$

Proof. Let $c \leq \mathrm{x}_{k+1, n}<x_{k, n} \leq d$. Then by (3.21), we see $\left|x_{k, n}-x_{k+1, n}\right| \sim \sqrt{a_{n}} / n$. 
Now, choose $\alpha, \beta>0$ satisfying for all $\mathrm{x}_{k+1, n}, x_{k, n} \in[c, d]$,

$$
\alpha \frac{\sqrt{a_{n}}}{n} \leq\left|x_{k, n}-x_{k+1, n}\right| \leq \beta \frac{\sqrt{a_{n}}}{n} .
$$

Let $x \in[a, b]$ and $\left.\left|x-{ }_{x j(x), n}\right|=\min \left\{\mid x-x_{k, n}\right\} \mid ; x_{k, n} \in[a, b]\right\}, x_{x j(c)+1, n}<c \leq_{x j(c), n}$, and $x j(d), n \leq d<_{x j(d)-1, n}$. Moreover, we take a non-negative integer $j_{k}$ satisfying for each $x_{k, n}$ $\in[a, b]$ and $k \neq j(x)$,

$$
\left(j_{k}+\frac{1}{2}\right) \alpha \frac{\sqrt{a_{n}}}{n} \leq\left|x-x_{k, n}\right| \leq\left(j_{k}+1\right) \beta \frac{\sqrt{a_{n}}}{n} .
$$

Then we have

$$
\begin{aligned}
& \max _{a \leq x \leq b} \sum_{c \leq x_{k, n} \leq d}\left|l_{k, n}^{m}(x) \sum_{i=0}^{m-2} e_{i}(m, k, n)\left(x-x_{k, n}\right)^{i}\right| \\
& \leq \max _{a \leq x \leq b} \sum_{i=0}^{m-2} \sum_{c \leq x_{k, n} \leq d}\left|\left(\frac{p_{n}\left(w_{\rho}^{2} ; x\right)}{\left(x-x_{k, n}\right) p_{n}^{\prime}\left(w_{\rho}^{2} ; x_{k, n}\right)}\right)^{m} e_{i}(m, k, n)\left(x-x_{k, n}\right)^{i}\right| \\
& \leq \max _{a \leq x \leq b} \sum_{i=0}^{m-2}\left[\left|\frac{p_{n}\left(w_{\rho}^{2} ; x\right)}{\left(x-x_{j(x), n}\right) p_{n}^{\prime}\left(w_{\rho}^{2} ; x_{j(x), n}\right)}\right|^{m}\left|e_{i}(m, k, n)\left(x-x_{j(x), n}\right)^{i}\right|\right. \\
& \left.\quad+\sum_{\substack{c \leq x_{k, n} \leq d \\
x_{k, n} * x_{j}(x), n}}\left|\left(\frac{p_{n}\left(w_{\rho}^{2} ; x\right)}{\left(x-x_{k, n}\right) p_{n}^{\prime}\left(w_{\rho}^{2} ; x_{k, n}\right)}\right)^{m} e_{i}(m, k, n)\left(x-x_{k, n}\right)^{i}\right|\right] .
\end{aligned}
$$

Here, by (3.16) and (3.17) we see

$$
\left|\frac{p_{n}\left(w_{\rho}^{2} ; x\right)}{\left(x-x_{j(x), n}\right) p_{n}^{\prime}\left(w_{\rho}^{2} ; x_{j(x), n}\right)}\right|^{m} \leq C
$$

and from (3.20), we have $|x j(x), n| \leq b+1$, and so by (1.6) we have

$$
\left|e_{i}(m, j(x), n)\left(x-x_{j(x), n}\right)^{i}\right| \leq C\left(\frac{n}{\sqrt{a_{2 n}-x_{j(x), n}}}\right)^{i} x_{j(x), n}^{-\frac{i}{2}}\left(\frac{\sqrt{a_{n}}}{n}\right)^{i} \leq C .
$$

Consequently, we have

$$
\left|\frac{p_{n}\left(w_{\rho^{2}}^{2} x\right)}{\left(x-x_{j(x), n}\right) p_{n}^{\prime}\left(w_{\rho}^{2} ; x_{j(x), n}\right)}\right|^{m}\left|e_{i}(m, j(x), n)\left(x-x_{j(x) n}\right)^{i}\right| \leq C .
$$

Similarly, for $c \leq x_{k, n} \leq d$ with $x_{k, n} \neq x_{x j(x), n}$, we have by (3.18) and (3.20),

$$
\left|\frac{p_{n}\left(w_{\rho}^{2} ; x\right)}{p_{n}^{\prime}\left(w_{\rho}^{2} ; x_{k, n}\right)}\right|^{m} \leq\left(\frac{\sqrt{a_{n}}}{n}\right)^{m}
$$

and by (1.6) and (3.23),

$$
\left|e_{i}(m, k, n)\left(x-x_{k, n}\right)^{i-m}\right| \leq C\left(\frac{n}{\sqrt{a_{n}}}\right)^{i}\left(\frac{n}{\sqrt{a_{n}}} \frac{1}{\left(j_{k}+1 / 2\right) \alpha}\right)^{m-i} .
$$


Therefore, we have for $0 \leq i \leq m-2$,

$$
\begin{aligned}
& \sum_{\substack{c \leq x_{k, n} \leq d \\
x_{k, n} \neq x_{j}(x), n}}\left|\left(\frac{p_{n}\left(w_{\rho}^{2} ; x\right)}{\left(x-x_{k, n}\right) p_{n}^{\prime}\left(w_{\rho}^{2} ; x_{k, n}\right)}\right)^{m} e_{i}(m, k, n)\left(x-x_{k, n}\right)^{i}\right| \\
& \leq C \sum_{\substack{c \leq x_{k, n} \leq d \\
x_{k, n} \neq x_{j}(x), n}}\left(\frac{1}{\left(j_{k}+1 / 2\right) \alpha}\right)^{2} \leq C .
\end{aligned}
$$

Therefore,

$$
\max _{a \leq x \leq b} \sum_{c \leq x_{k, n} \leq d}\left|l_{k, n}^{m}(x) \sum_{i=0}^{m-2} e_{i}(m, k, n)\left(x-x_{k, n}\right)^{i}\right| \leq C .
$$

Proof of Theorem 1.10. We use Theorem 1.9 and Lemma 3.11. We find a lower bound for the Lebesgue constants $\lambda_{n}(m,[a, b])=\max _{a \leq x \leq b} \sum_{k=1}^{n}\left|h_{k n}(m ; x)\right|$ with a positive odd order $m$ and a given interval $[a, b], 0<a<b<\infty$. By the expression (1.4) we have

$$
\begin{aligned}
\left|h_{k n}(m ; x)\right| \geq\left|l_{k, n}^{m}(x) e_{m-1}(m, k, n)\left(x-x_{k, n}\right)^{m-1}\right| & \\
& -\left|l_{k, n}^{m}(x) \sum_{i=0}^{m-2} e_{i}(m, k, n)\left(x-x_{k, n}\right)^{i}\right| .
\end{aligned}
$$

Let $c=a / 2, d=b+(b-a)$ and

$$
\begin{aligned}
\lambda_{n}(m,[a, b]) \geq & \max _{a \leq x \leq b} \sum_{c \leq x_{k, n} \leq d}\left|l_{k, n}^{m}(x) e_{m-1}(m, k, n)\left(x-x_{k, n}\right)^{m-1}\right| \\
& -\max _{a \leq x \leq b} \sum_{c \leq x_{k, n} \leq d}\left|l_{k, n}^{m}(x) \sum_{i=0}^{m-2} e_{i}(m, k, n)\left(x-x_{k, n}\right)^{i}\right| \\
= & \max _{a \leq x \leq b} F_{n}(x)-\max _{a \leq x \leq b} G_{n}(x) .
\end{aligned}
$$

It follows from Lemma 3.11 that $\max _{a} \leq x \leq b G_{n}(x) \leq C$ with $C$ independent of $n$. Therefore, it is enough to show that $\max _{a \leq x \leq b} F_{n}(x) \geq C \log (1+n)$. We consider $\alpha$, $\beta$ and $j_{k}$ defined in (3.22) and (3.23). Let $K(x ;[c, d])$ be the set of numbers defined as

$$
K(x ;[c, d])=\left\{j_{k} ;\left(j_{k}+1 / 2\right) \alpha \frac{\sqrt{a_{n}}}{n} \leq\left|x-x_{k, n}\right| \leq\left(j_{k}+1\right) \beta \frac{\sqrt{a_{n}}}{n}, x_{k, n} \in[c, d], k \neq j(x)\right\},
$$

where $j_{k}$ is a non-negative integer. Then, there exist $\gamma>0$ and $C>0$ such that

$$
C n^{\gamma} \leq \max \left\{j_{k} \in K(x ;[c, d])\right\},
$$

that is, we see

$$
\left\{0,1,2, \ldots,\left[C n^{\gamma}\right]\right\} \subset K(x ;[c, d]) .
$$

In fact, from Lemma 3.10 (f), we see $a_{n} \leq c_{1} n^{1 / \Lambda}, \Lambda>1 / 2$. By (3.22) and (3.23), we see $0 \in K(x ;[c, d])$ and

$$
\left(\max \left\{j_{k} \in K(x ;[c, d])\right\}+1\right) \beta \frac{\sqrt{a_{n}}}{n} \geq b-a .
$$


Hence, we have

$$
\max \left\{j_{k} \in K(x ;[c, d])\right\}+1 \geq \frac{b-a}{\beta} \frac{n}{\sqrt{a_{n}}} \geq \frac{b-a}{\beta} \frac{n}{\sqrt{a_{n}}} \frac{1}{\sqrt{c_{1}}} n^{1-1 /(2 \Lambda)}=: C n^{\gamma} .
$$

So, we have (3.24). Now, we take an interval $\left[x_{l+1, n}, x_{l, n}\right] \subset[a, b]$, and we put

$$
x^{*}:=\left(x_{\ell+1, n}+x_{\ell, n}\right) / 2 .
$$

By Lemma 3.10 (d), we have

$$
\left|\left(p_{n} w_{\rho}\right)\left(x^{*}\right)\right| \sim \frac{1}{a_{n}^{1 / 4}}
$$

From Lemma 3.10 (e), we know for $c \leq x_{k, n} \leq d$

$$
\left|x^{*}-x_{k, n}\right| \geq \frac{\alpha}{2} \frac{\sqrt{a_{n}}}{n}
$$

By Lemma 3.10 (c), we have for $c \leq x_{k, n} \leq d$

$$
\left|p_{n}^{\prime}\left(x_{k, n}\right)\right| w_{\rho}\left(x_{k, n}\right) \sim \frac{n}{a_{n}^{3 / 4}} .
$$

Then, we have

$$
\begin{aligned}
\left|l_{k, n}\left(x^{*}\right)\right| & =\frac{\left|p_{n}\left(x^{*}\right)\right| w\left(x^{*}\right)\left(x^{*}+\frac{a_{n}}{n^{2}}\right)^{\rho}}{\left|\left(x^{*}-x_{k, n}\right) p_{n}^{\prime}\left(x_{k, n}\right)\right| w_{\rho}\left(x_{k, n}\right)} \frac{w_{\rho}\left(x_{k, n}\right)}{w\left(x^{*}\right)\left(x^{*}+\frac{a_{n}}{n^{2}}\right)^{\rho}} \\
& \geq C \frac{\sqrt{a_{n}}}{n} \frac{1}{\left|x^{*}-x_{k, n}\right|} .
\end{aligned}
$$

Here, we used the following facts:

$$
\left(x^{*}+\frac{a_{n}}{n}\right)^{\rho} \sim x^{* \rho}
$$

and

$$
\frac{w_{\rho}\left(x_{k, n}\right)}{w_{\rho}\left(x^{*}\right)} \sim 1, \quad x^{*}, x_{k, n} \in[a, b] .
$$

Now we use Theorem 1.9, that is, for $c \leq x_{k, n} \leq d$ we have

$$
e_{m-1}(m, k, n) \sim\left(\frac{n}{\sqrt{a_{n}}}\right)^{m-1} .
$$

Therefore, with (3.25) and (3.26), we have

$$
\begin{aligned}
F_{n}\left(x^{*}\right) & \geq \sum_{j_{k} \in K\left(x^{*} ;[c, d]\right)}\left|l_{k, n}^{m}\left(x^{*}\right) e_{m-1}(m, k, n)\left(x^{*}-x_{k, n}\right)^{m-1}\right| \\
& \geq C \sum_{j_{k} \in K\left(x^{*} ;[c, d]\right)}\left(\frac{\sqrt{a_{n}}}{n} \frac{1}{\left|x^{*}-x_{k, n}\right|}\right)^{m}\left(\frac{n}{\sqrt{a_{n}}}\right)^{m-1}\left|x^{*}-x_{k, n}\right|^{m-1} \\
& =C \sum_{j_{k} \in K\left(x^{*} ;[c, d]\right)}\left(\frac{\sqrt{a_{n}}}{n}\right)^{m} \frac{1}{\left|x^{*}-x_{k, n}\right|}\left(\frac{n}{\sqrt{a_{n}}}\right)^{m-1} \\
& \geq C \sum_{j_{k} \in K\left(x^{*} ;[c, d]\right)}\left(\frac{\sqrt{a_{n}}}{n}\right)^{m} \frac{1}{\left(j_{k}+1\right) \beta\left(\sqrt{a_{n}} / n\right)}\left(\frac{n}{\sqrt{a_{n}}}\right)^{m-1} \\
& \geq C(\beta) \sum_{j_{k} \in K\left(x^{*} ;[c, d]\right)} \frac{1}{j_{k}+1} \geq C \sum_{0 \leq j \leq n^{\gamma}} \frac{1}{j+1} \geq C \log n .
\end{aligned}
$$


Consequently, the theorem is complete.

\section{Acknowledgements}

The authors thank the referees for many valuable suggestions and comments. Hee Sun Jung was supported by SEOK CHUN Research Fund, Sungkyunkwan University, 2010.

\section{Author details}

${ }^{1}$ Department of Mathematics Education, Sungkyunkwan University Seoul 110-745, Republic of Korea ${ }^{2}$ Department of Mathematics, Meijo University Nagoya 468-8502, Japan

\section{Authors' contributions}

All authors conceived of the study, participated in its design and coordination, drafted the manuscript, participated in the sequence alignment. All authors read and approved the final manuscript.

\section{Competing interests}

The authors declare that they have no competing interests.

Received: 28 July 2011 Accepted: 25 November 2011 Published: 25 November 2011

\section{References}

1. Jung, HS, Sakai, R: Some properties of orthonormal polynomials for Laguerre-type weights. J Inequal Appl 2011, 25 (2011). (Article ID 372874). doi:10.1186/1029-242X-2011-25

2. Jung, $\mathrm{HS}$, Sakai, R: Derivatives of orthonormal polynomials and coefficients of Hermite-Fejér interpolation polynomial with exponential-type weights. J Inequal Appl 2010, 29 (2010). (Article ID 816363)

3. Jung, HS, Sakai, R: Markov-Bernstein inequality and Hermite-Fejér interpolation for exponential-type weights. J Approx Theory. 162, 1381-1397 (2010). doi:10.1016/j.jat.2010.02.006

4. Kanjin, Y, Sakai, R: Pointwise convergence of Hermite-Fejér interpolation of higher order for Freud weights. Tohoku Math J. 46, 181-206 (1994). doi:10.2748/tmj/1178225757

5. Levin, AL, Lubinsky, DS: Orthogonal polynomials for exponential weights $x^{2 \rho} e^{-2 Q(x)}$ on $[0, d)$, II. J Approx Theory. 139, 107-143 (2006). doi:10.1016/j.jat.2005.05.010

6. Levin, AL, Lubinsky, DS: Orthogonal polynomials for exponential weights $x^{2 \rho} e^{-2 Q(x)}$ on [0, d). J Approx Theory. 134, 199-256 (2005). doi:10.1016/j.jat.2005.02.006

7. Jung, HS, Sakai, R: Specific examples of exponential weights. Commun Korean Math Soc. 24(2), 303-319 (2009). doi:10.4134/CKMS.2009.24.2.303

8. Freud, G: Orthogonal Polynomials. Pergamon Press, Oxford (1971)

9. Sakai, R, Vértesi, P: Hermite-Fejér interpolations of higher order III. Studia Sci Math Hungarica. 28, 87-97 (1993)

10. Levin, AL, Lubinsky, DS: Orthogonal Polynomials for Exponential Weights. Springer, New York (2001)

11. Jung, HS, Sakai, R: Derivatives of integrating functions for orthonormal polynomials with exponential-type weights. J Inequal Appl 2009, 22 (2009). (Article ID 528454)

12. Jung, HS, Sakai, R: Inequalities with exponential weights. J Comput Appl Math. 212, 359-373 (2008). doi:10.1016/j. cam.2006.12.011

13. Jung, HS, Sakai, R: Orthonormal polynomials with exponential-type weights. J Approx Theory. 152, 215-238 (2008). doi:10.1016/j.jat.2007.12.004

14. Kasuga, T, Sakai, R: Orthonormal polynomials for generalized Freud-type weights. J Approx Theory. 121, 13-53 (2003). doi:10.1016/50021-9045(02)00041-2

doi:10.1186/1029-242X-2011-122

Cite this article as: Jung and Sakai: Higher order Hermite-Fejér interpolation polynomials with Laguerre-type weights. Journal of Inequalities and Applications 2011 2011:122.

\section{Submit your manuscript to a SpringerOpen ${ }^{\circ}$ journal and benefit from:}

- Convenient online submission

- Rigorous peer review

- Immediate publication on acceptance

- Open access: articles freely available online

- High visibility within the field

- Retaining the copyright to your article

Submit your next manuscript at $\boldsymbol{s p r i n g e r o p e n . c o m ~}$ 